\title{
Retinal thickness after vitrectomy and internal limiting membrane peeling for macular hole and epiretinal membrane
}

This article was published in the following Dove Press journal:

Clinical Ophthalmology

3 May 2012

Number of times this article has been viewed

\section{Kazuyuki Kumagai' \\ Nobuchika Ogino' \\ Mariko Furukawa ${ }^{2}$ \\ Masanori Hangai ${ }^{3}$ \\ Shigeyasu Kazama' \\ Shirou Nishigaki ${ }^{4}$ \\ Eric Larson ${ }^{5}$}

'Shinjo Ophthalmologic Institute, Miyazaki, ${ }^{2}$ Department of

Ophthalmology, Kami-iida First General Hospital, Nagoya,

${ }^{3}$ Department of Ophthalmology and Visual Sciences, Kyoto University

Graduate School of Medicine, Kyoto, ${ }^{4}$ Nishigaki Eye Clinic, Nagoya, ${ }^{5}$ Miyazaki Prefectural Nursing

University, Miyazaki, Japan
Correspondence: Kazuyuki Kumagai Shinjo Ophthalmologic Institute, 889-I Mego Shimokitakata-cho, Miyazaki 880-035, Japan

Tel $+8 \mid 985294700$

Fax +8I 985258095

Email ganka@kamiiida-hp.jp
Purpose: To determine the retinal thickness (RT), after vitrectomy with internal limiting membrane (ILM) peeling, for an idiopathic macular hole $(\mathrm{MH})$ or an epiretinal membrane (ERM). Also, to investigate the effect of a dissociated optic nerve fiber layer (DONFL) appearance on RT.

Methods: A non-randomized, retrospective chart review was performed for 159 patients who had successful closure of a MH, with $(n=148)$, or without $(n=11)$, ILM peeling. Also studied were 117 patients who had successful removal of an ERM, with $(n=104)$, or without ( $\mathrm{n}=13$ ), ILM peeling. The RT of the nine Early Treatment Diabetic Retinopathy Study areas was measured by spectral domain optical coherence tomography (SD-OCT). In the MH-withILM peeling and ERM-with-ILM peeling groups, the RT of the operated eyes was compared to the corresponding areas of normal fellow eyes. The inner temporal/inner nasal ratio (TNR) was used to assess the effect of ILM peeling on RT. The effects of DONFL appearance on RT were evaluated in only the MH-with-ILM peeling group.

Results: In the MH-with-ILM peeling group, the central, inner nasal, and outer nasal areas of the retina of operated eyes were significantly thicker than the corresponding areas of normal fellow eyes. In addition, the inner temporal, outer temporal, and inner superior retina was significantly thinner than in the corresponding areas of normal fellow eyes. Similar findings were observed regardless of the presence of a DONFL appearance. In the ERM-with-ILM peeling group, the retina of operated eyes was significantly thicker in all areas, except the inner and outer temporal areas. In the MH-with-ILM peeling group, the TNR was 0.86 in operated eyes, and 0.96 in fellow eyes $(P<0.001)$. In the ERM-with-ILM peeling group, the TNR was 0.84 in operated eyes, and 0.95 in fellow eyes $(P<0.001)$. TNR in operated eyes of the MH-without-ILM peeling group was 0.98 , which was significantly greater than that of the MH-with-ILM peeling group $(P<0.001)$. TNR in the operated eyes of the ERM-without-ILM peeling group was 0.98 , which was significantly greater than that of ERM-with-ILM peeling group $(P<0.001)$.

Conclusion: The thinning of the temporal retina and thickening of the nasal retina after ILM peeling does not appear to be disease-specific. In addition, changes in RT after ILM peeling are not related to the presence of a DONFL appearance.

Keywords: epiretinal membrane, macular hole, optical coherence tomography, retinal thickness, internal limiting membrane

\section{Introduction}

Vitrectomy with internal limiting membrane (ILM) peeling is used to treat eyes with a macular hole $(\mathrm{MH})^{1-7}$ or an epiretinal membrane (ERM).$^{8-14}$ In MH surgery, ILM peeling has become a standard procedure, because of a high rate of successful closure and low reopening rate. In ERM surgery, ILM peeling results in a disappearance 
of retinal folds, ${ }^{8}$ reduced recurrences, ${ }^{9,10}$ and disappearance of pseudoholes. ${ }^{11}$

The adverse effects of ILM peeling include damage to the functioning of Müller cells, ${ }^{1}$ selective delay in recovery of the b-wave of focal macular electroretinograms, ${ }^{15}$ and decreased retinal sensitivity. ${ }^{12}$ Only a few studies have investigated the effect of ILM peeling on retinal thickness (RT). ${ }^{5,7,12-14,16}$ It has been reported that the foveal contour is asymmetrical in horizontal optical coherence tomographic (OCT) images of eyes in which MHs were successfully closed by vitrectomy with ILM peeling. ${ }^{17}$ The mechanism that causes this asymmetrical foveal contour after MH surgery with ILM peeling has not been determined. However, we have noted that this asymmetrical contour is also observed after ERM surgery.

Another change in the appearance of the fundus after ILM peeling is the appearance of a dissociated optic nerve fiber layer (DONFL). DONFL appearance is characterized by numerous arcuate retinal striae running parallel to optic nerve fibers in the macular area, and can be seen via conventional ophthalmoscopic examination. DONFL appearance is considered to be related to ILM peeling, and has no adverse effect on the function of the retina. ${ }^{8,18-25}$ DONFL appearance is reported to be caused by dimples on the retina, their depth limited to the thickness of the retinal nerve fiber layer. ${ }^{22}$

The purpose of this study was to determine whether alterations of RT, detected after a closure of an idiopathic MH by vitrectomy with ILM peeling, are also present after vitrectomy with ILM peeling for an ERM. In addition, we aimed to investigate whether the alterations of RT were related to the presence of a DONFL appearance.

\section{Methods}

A non-randomized, retrospective chart review was performed for 276 eyes of 276 patients with successful vitrectomy, (with and without ILM peeling) for an idiopathic MH or idiopathic ERM. The surgeries were performed between May 1994 and July 2008. The patients included 188 women (68.1\%) and 88 men $(31.9 \%)$. Their mean age was $68.2 \pm 7.9$ years, with a range from 35 to 86 years.

Eyes were excluded if they had refractive errors (spherical equivalent $<-6$ diopters), reoperation for postoperative complications (such as reopening of the $\mathrm{MH}$ or retinal detachment), poor OCT images, other ocular pathology that could affect the retinal thickness (such as glaucoma and other optic nerve diseases), and age-related macular degeneration. The fellow eyes were normal and did not have any macular disease, glaucoma, or any subclinical abnormal tomographic features (such as vitreofoveal traction or residual foveal deformation). ${ }^{26}$

The research adhered to the tenets set forth in the Declaration of Helsinki. Approval for data collection and analysis was obtained from the institutional review board. Written, informed consent was obtained from all patients.

Subjects were divided into four groups, based on their disease and whether ILM was intentionally performed: $\mathrm{MH}$ with-ILM peeling group $(\mathrm{n}=148)$; MH-without-ILM peeling group $(n=11)$; ERM-with-ILM peeling group $(n=104)$; and ERM-without-ILM peeling group $(n=13)$. In MH surgery, ILM peeling was performed in all eyes after March, 1998, and not performed before this time. In ERM surgery, ILM peeling was performed in a large proportion of eyes; in a small number of cases with weak retinal wrinkling, ILM peeling was not performed.

All surgeries were performed by one surgeon: Nobuchika Ogino. All phakic patients, except one 35-year-old patient, underwent pars plana vitrectomy with phacoemulsification and placement of a posterior chamber intraocular lens, to avoid postoperative nuclear cataract progression. After the removal of vitreous gel and the posterior hyaloid, the ERM was peeled from the macular surface using membrane forceps. ILM peeling was performed without staining in eight eyes. In 244 eyes, triamcinolone acetonide was used to stain the ILM. ${ }^{27}$ ILM peeling was started by grasping the ILM over the superior macular region, with forceps, and peeling the ILM over two to three disc diameters around the fovea. In MH surgery, a sulfur hexafluoride gas tamponade was used in all cases, and patients were instructed to maintain a prone position for 7 days. At the time of surgery, posterior vitreous detachment was present in 37 eyes $(23 \%)$ in $\mathrm{MH}$ groups, and 109 eyes (93\%) in ERM groups.

We used the retinal thickness map analysis protocol of Cirrus $^{\mathrm{TM}}$ OCT (Carl Zeiss Meditec, Dublin, CA). The Cirrus OCT macular images were acquired for a macular cube of $200 \times 200$ pixels, using the 5 -line raster scanning protocol. All scans were obtained by experienced OCT examiners. The scans were taken three times to obtain images with the highest signal intensity (ie, signal strength $>7$ ). Scans with centering errors or minimal segmentation errors were excluded.

RT maps show the average RT in each of nine macular subfields in a $6 \mathrm{~mm}$ diameter circle, centered on the fovea, as defined in the Early Treatment Diabetic Retinopathy Study (ETDRS). ${ }^{28}$ The standard retinal subfields were the central, and the superior, temporal, inferior, and nasal quadrants of the inner and outer rings. The diameter of the central ring was $1 \mathrm{~mm}$, that of the inner ring was $3 \mathrm{~mm}$, and that of the 
outer ring was $6 \mathrm{~mm}$. The software in the Cirrus package automatically calculated the average retinal thickness within each ETDRS subfield, by averaging retinal thickness in the inner ring (at 1416 sampling points in the central subfield, 2826 sampling points in the superior and inferior subfields, and 2842 sampling points of the temporal and nasal subfields), and 9544 sampling points in each of four subfields of the outer ring.

Postoperative RT was measured at least 12 months postoperatively. The mean interval between surgery and measurement was $38.9 \pm 29.8$ months (range: 12-168) for the MH-with-ILM peeling group, $141.4 \pm 69.4$ months (range: 12-201) for the MH-without-ILM peeling group, $24.6 \pm 16.7$ months (range: 12-81) for the ERM-with-ILM peeling group, and $26.9 \pm 12.4$ months (range: 12-48) for the ERM-without-ILM peeling group.

For the MH-with-ILM peeling and ERM-with-ILM peeling groups, the RT of the operated eyes was compared to the corresponding areas of normal fellow eyes. Because there were only 14 normal fellow eyes in the group without ILM peeling, we used the temporal to nasal ratio (TNR) to compare the temporal-nasal differences in eyes with and without ILM peeling.

A clearly visible DONFL was determined on fundus photographs taken at least 3 months after surgery. The relationship between DONFL appearance and RT was evaluated in only the MH-with-ILM peeling group.

Numerical data were analyzed by paired and unpaired $t$-tests. A $P$ value $<0.05$ was considered statistically significant. Statistical analyses of the data were carried out using Statview software (Abacus Concepts, Berkeley, CA).

\section{Results}

The RTs of nine OCT areas of operated eyes, and their fellow eyes, in the MH-with-ILM peeling group are shown in Table 1. The central, inner nasal, and outer nasal areas in operated eyes were significantly thicker than the corresponding areas of fellow eyes. By contrast, the inner temporal, inner superior, and outer temporal areas in operated eyes were significantly thinner than those of fellow eyes.

The RTs of nine OCT areas in the ERM-with-ILM peeling group, and their fellow eyes, are shown in Table 2. The RTs of all subfields in the operated eyes were significantly greater than those of fellow eyes, except in the inner and outer temporal areas.

The RTs of nine OCT areas after MH surgery, with and without ILM peeling, are shown in Table 3. The retina in the central and inner nasal areas in the MH-with-ILM peeling
Table I Retinal thickness of operated and normal fellow eyes after MH surgery with ILM peeling

\begin{tabular}{|c|c|c|c|}
\hline $\begin{array}{l}\text { ETDRS } \\
\text { subfield }\end{array}$ & $\begin{array}{l}\text { Operated eyes } \\
(n=148)\end{array}$ & $\begin{array}{l}\text { Normal fellow eyes } \\
(n=148)\end{array}$ & $P$-value \\
\hline Central & $293 \pm 36.3$ & $24 I \pm 2 I .4$ & $<0.001$ \\
\hline Inner superior & $317 \pm 20.9$ & $32 I \pm 15.5$ & 0.01 \\
\hline Inner temporal & $295 \pm 20.3$ & $309 \pm 15.5$ & $<0.001$ \\
\hline Inner inferior & $318 \pm 19.4$ & $317 \pm 15.7$ & 0.71 \\
\hline Inner nasal & $344 \pm 20.3$ & $322 \pm 20.3$ & $<0.001$ \\
\hline Outer superior & $278 \pm 17.3$ & $278 \pm 14.7$ & 0.58 \\
\hline Outer temporal & $259 \pm 17.8$ & $262 \pm 13.5$ & 0.01 \\
\hline Outer inferior & $268 \pm 17.1$ & $266 \pm 16.7$ & 0.31 \\
\hline Outer nasal & $299 \pm 18.4$ & $294 \pm 19.6$ & 0.002 \\
\hline
\end{tabular}

Note: Data shown are means \pm standard deviations.

Abbreviations: $\mathrm{MH}$, macular hole; ILM, internal limiting membrane peeling; ETDRS, early treatment diabetic retinopathy study.

group was significantly thicker than in the group without ILM peeling, whereas the retina in the inner temporal area was significantly thinner. There were no statistically significant differences $(P=0.24-0.92)$ in RTs between the right and left eyes after MH surgery with ILM peeling.

The RTs of nine OCT areas after ERM surgery, with and without ILM peeling, are shown in Table 4. The retina in the inner temporal and outer temporal areas of the ERMwith-ILM peeling group was significantly thinner than in the group with no ILM peeling. The central $(P=0.10)$ and inner $(P=0.07)$ nasal RTs were greater than those of the group without ILM peeling, but the differences were not significant. There were no statistically significant differences $(P=0.16-0.81)$ in RTs between the right and left eyes after ERM surgery with ILM peeling.

In eyes with ERM, the RT of the preoperative macular area was greater than that of normal eyes, making it difficult to determine whether asymmetrical thicknesses were present

Table 2 Retinal thickness of operated and normal fellow eyes after EMR surgery with ILM peeling

\begin{tabular}{|c|c|c|c|}
\hline $\begin{array}{l}\text { ETDRS } \\
\text { subfield }\end{array}$ & $\begin{array}{l}\text { Operated eyes } \\
(n=104)\end{array}$ & $\begin{array}{l}\text { Normal fellow eyes } \\
(n=104)\end{array}$ & $P$-value \\
\hline Central & $354 \pm 54.2$ & $254 \pm 24.7$ & $<0.001$ \\
\hline Inner superior & $341 \pm 37.5$ & $325 \pm 17.6$ & $<0.001$ \\
\hline Inner temporal & $316 \pm 36.3$ & $314 \pm 16.1$ & 0.50 \\
\hline Inner inferior & $337 \pm 33.4$ & $321 \pm 17.4$ & $<0.001$ \\
\hline Inner nasal & $375 \pm 39.3$ & $329 \pm 17.9$ & $<0.001$ \\
\hline Outer superior & $284 \pm 25.8$ & $279 \pm 17.5$ & 0.04 \\
\hline Outer temporal & $267 \pm 23.0$ & $264 \pm 14.3$ & 0.16 \\
\hline Outer inferior & $272 \pm 25.7$ & $267 \pm 14.5$ & 0.03 \\
\hline Outer nasal & $308 \pm 32.4$ & $297 \pm 18.3$ & $<0.001$ \\
\hline
\end{tabular}

Note: Data shown are means \pm standard deviations.

Abbreviations: ERM, epiretinal membrane; ILM, internal limiting membrane peeling; ETDRS, early treatment diabetic retinopathy study. 
Table 3 Retinal thickness after $\mathrm{MH}$ surgery with and without ILM peeling

\begin{tabular}{|c|c|c|c|}
\hline $\begin{array}{l}\text { ETDRS } \\
\text { subfield }\end{array}$ & $\begin{array}{l}\text { ILM peeling (+) } \\
(\mathrm{n}=148)\end{array}$ & $\begin{array}{l}\text { ILM peeling (-) } \\
(\mathrm{n}=\mathrm{II})\end{array}$ & $P$-value \\
\hline Central & $293 \pm 36.3$ & $24 I \pm 20 . I$ & $<0.001$ \\
\hline Inner superior & $317 \pm 20.9$ & $316 \pm 31.1$ & 0.95 \\
\hline Inner temporal & $295 \pm 20.3$ & $311 \pm 30.4$ & 0.02 \\
\hline Inner inferior & $318 \pm 19.4$ & $310 \pm 33.7$ & 0.25 \\
\hline Inner nasal & $344 \pm 20.3$ & $318 \pm 31.5$ & $<0.001$ \\
\hline Outer superior & $278 \pm 17.3$ & $274 \pm 27.9$ & 0.51 \\
\hline Outer temporal & $259 \pm 17.8$ & $263 \pm 24.4$ & 0.45 \\
\hline Outer inferior & $268 \pm 17.1$ & $267 \pm 24.3$ & 0.95 \\
\hline Outer nasal & $299 \pm 18.4$ & $290 \pm 28.6$ & 0.14 \\
\hline
\end{tabular}

Note: Data shown are means \pm standard deviations.

Abbreviations: $\mathrm{MH}$, macular hole; ILM, internal limiting membrane peeling; ETDRS, early treatment diabetic retinopathy study.

after surgery. Therefore, we calculated TNR to represent temporal-nasal differences in thickness. In the MH-withILM peeling group, TNR was $0.86 \pm 0.051$ in operated eyes, and $0.96 \pm 0.051$ in fellow eyes $(P<0.001)$. In the ERM-with-ILM peeling group, TNR was $0.84 \pm 0.072$ in operated eyes, and $0.95 \pm 0.036$ in fellow eyes $(P<0.001)$. TNR in the operated eyes of the MH-without-ILM peeling group was $0.98 \pm 0.035$, which was significantly greater than that of the MH-with-ILM peeling group $(P<0.001)$. TNR in operated eyes of the ERM-without-ILM peeling group was $0.98 \pm 0.074$, which was significantly greater than in the ERM-with-ILM peeling group $(P<0.001)$.

A clearly visible DONFL was observed in 92 (62\%) of the 148 eyes in the MH-with-ILM peeling group. The RTs of the nine ETDRS areas in operated eyes, and their fellow eyes, with and without a DONFL appearance, after $\mathrm{MH}$ surgery with ILM peeling, are shown in Tables 5 and 6 . A thinner inner temporal area and a thicker inner nasal area

Table 4 Retinal thickness after ERM surgery with and without ILM peeling

\begin{tabular}{|c|c|c|c|}
\hline $\begin{array}{l}\text { ETDRS } \\
\text { subfield }\end{array}$ & $\begin{array}{l}\text { ILM peeling }(+) \\
(n=104)\end{array}$ & $\begin{array}{l}\text { ILM peeling }(-) \\
(n=13)\end{array}$ & $P$-value \\
\hline Central & $354 \pm 54.2$ & $326 \pm 72.9$ & 0.1 \\
\hline Inner superior & $341 \pm 37.5$ & $343 \pm 16.0$ & 0.86 \\
\hline Inner temporal & $316 \pm 36.3$ & $348 \pm 22.1$ & 0.003 \\
\hline Inner inferior & $337 \pm 33.4$ & $335 \pm 21.7$ & 0.83 \\
\hline Inner nasal & $375 \pm 39.3$ & $355 \pm 19.9$ & 0.07 \\
\hline Outer superior & $284 \pm 25.8$ & $294 \pm 25.1$ & 0.17 \\
\hline Outer temporal & $267 \pm 23.0$ & $286 \pm 33.3$ & 0.01 \\
\hline Outer inferior & $272 \pm 25.7$ & $270 \pm 27.0$ & 0.78 \\
\hline Outer nasal & $308 \pm 32.4$ & $304 \pm 18.6$ & 0.71 \\
\hline
\end{tabular}

Note: Data shown are means \pm standard deviations.

Abbreviations: ERM, epiretinal membrane; ILM, internal limiting membrane peeling; ETDRS, early treatment diabetic retinopathy study.
Table 5 Retinal thickness of operated and normal fellow eyes with a DONFL appearance after macular hole surgery with ILM peeling

\begin{tabular}{|c|c|c|c|}
\hline $\begin{array}{l}\text { ETDRS } \\
\text { subfield }\end{array}$ & $\begin{array}{l}\text { Operated eyes } \\
(n=92)\end{array}$ & $\begin{array}{l}\text { Normal fellow eyes } \\
(n=92)\end{array}$ & $P$-value \\
\hline Central & $297 \pm 35.2$ & $239 \pm 22.0$ & $<0.001$ \\
\hline Inner superior & $320 \pm 19.1$ & $321 \pm 14.7$ & 0.72 \\
\hline Inner temporal & $298 \pm 18.3$ & $308 \pm 15.5$ & $<0.001$ \\
\hline Inner inferior & $321 \pm 17.6$ & $317 \pm 15.9$ & 0.04 \\
\hline Inner nasal & $347 \pm 19.1$ & $321 \pm 21.0$ & $<0.001$ \\
\hline Outer superior & $28 I \pm 15.4$ & $280 \pm 14.1$ & 0.81 \\
\hline Outer temporal & $262 \pm 16.2$ & $264 \pm 12.8$ & 0.18 \\
\hline Outer inferior & $270 \pm 15.9$ & $267 \pm 16.0$ & 0.06 \\
\hline Outer nasal & $301 \pm 16.9$ & $294 \pm 21.9$ & 0.002 \\
\hline
\end{tabular}

Note: Data shown are means \pm standard deviations.

Abbreviations: ILM, internal limiting membrane; DONFL, dissociated optic nerve fiber layer; ETDRS, Early Treatment Diabetic Retinopathy Study.

were found in operated eyes of both groups, with or without a DONFL appearance.

The RTs of the nine ETDRS areas of eyes, with and without a DONFL appearance, are shown in Table 7. All areas of eyes with a DONFL appearance, except the central and inner nasal areas, were significantly thicker than those of eyes without a DONFL appearance.

\section{Discussion}

We measured the RTs of the nine ETDRS areas in 276 patients who had successful surgery for idiopathic $\mathrm{MH}$ or idiopathic ERM, with and without ILM peeling. Because macular thickness varies significantly with gender and age, ${ }^{29}$ we compared the RTs of operated eyes to the corresponding areas of normal fellow eyes.

In eyes with an $\mathrm{MH}$, we found that the temporal retina was significantly thinner, and the nasal retina significantly

Table 6 Retinal thickness of operated and normal fellow eyes without a DONFL appearance after macular hole surgery with ILM peeling

\begin{tabular}{|c|c|c|c|}
\hline $\begin{array}{l}\text { ETDRS } \\
\text { subfield }\end{array}$ & $\begin{array}{l}\text { Operated eyes } \\
(n=56)\end{array}$ & $\begin{array}{l}\text { Normal fellow eyes } \\
(n=56)\end{array}$ & $P$-value \\
\hline Central & $286 \pm 37.3$ & $244 \pm 20.2$ & $<0.001$ \\
\hline Inner superior & $311 \pm 22.6$ & $321 \pm 16.9$ & $<0.001$ \\
\hline Inner temporal & $288 \pm 21.9$ & $310 \pm 15.6$ & $<0.001$ \\
\hline Inner inferior & $312 \pm 21.0$ & $317 \pm 15.3$ & 0.05 \\
\hline Inner nasal & $340 \pm 21.7$ & $323 \pm 19.2$ & $<0.001$ \\
\hline Outer superior & $273 \pm 19.5$ & $276 \pm 15.4$ & 0.28 \\
\hline Outer temporal & $254 \pm 19.5$ & $259 \pm 14.3$ & 0.03 \\
\hline Outer inferior & $263 \pm 18.2$ & $264 \pm 17.7$ & 0.49 \\
\hline Outer nasal & $295 \pm 20.1$ & $293 \pm 15.2$ & 0.41 \\
\hline
\end{tabular}

Note: Data shown are means \pm standard deviations.

Abbreviations: ILM, internal limiting membrane; DONFL, dissociated optic nerve fiber layer; ETDRS, Early Treatment Diabetic Retinopathy Study. 
Table 7 Retinal thickness in eyes with and without a DONFL appearance after macular hole surgery with ILM peeling

\begin{tabular}{|c|c|c|c|}
\hline $\begin{array}{l}\text { ETDRS } \\
\text { subfield }\end{array}$ & $\begin{array}{l}\text { DONFL presence } \\
(n=92)\end{array}$ & $\begin{array}{l}\text { DONFL absence } \\
(n=56)\end{array}$ & $P$-value \\
\hline Central & $297 \pm 35.2$ & $286 \pm 37.3$ & 0.07 \\
\hline Inner superior & $320 \pm 19.1$ & $311 \pm 22.6$ & 0.01 \\
\hline Inner temporal & $298 \pm 18.3$ & $288 \pm 21.9$ & 0.003 \\
\hline Inner inferior & $321 \pm 17.6$ & $312 \pm 21.0$ & 0.006 \\
\hline Inner nasal & $347 \pm 19.1$ & $340 \pm 21.7$ & 0.06 \\
\hline Outer superior & $28 I \pm 15.4$ & $273 \pm 19.4$ & 0.01 \\
\hline Outer temporal & $262 \pm 16.2$ & $254 \pm 19.5$ & 0.02 \\
\hline Outer inferior & $270 \pm 15.9$ & $263 \pm 18.2$ & 0.007 \\
\hline Outer nasal & $301 \pm 16.9$ & $295 \pm 20.1$ & 0.046 \\
\hline
\end{tabular}

Note: Data shown are means \pm standard deviations.

Abbreviations: ILM, internal limiting membrane; DONFL, dissociated optic nerve fiber layer; ETDRS, Early Treatment Diabetic Retinopathy Study.

thicker, in eyes that had undergone vitrectomy with ILM peeling. This characteristic region-specific difference in macular thicknesses was found when the RTs of ILM-peeled eyes were compared to those of their fellow eyes, and when the comparison was made between eyes with and without ILM peeling. Region-specific significant differences in RTs between eyes with and without ILM peeling were also found after ERM surgery. TNR was significantly different between eyes that had, or had not, received ILM peeling after MH or ERM surgery. Taken together, our data suggest that the region-specific retinal thickness changes observed resulted from ILM peeling.

To further understand structural changes in the macula after surgery, color fundus photographs and Cirrus OCT images of two cases that underwent vitrectomy, with and without ILM peeling, for bilateral macular holes are shown in Figures 1 and 2. These eyes were not included in the analysis. We found significantly different macular features between ILM-peeled and non-ILM-peeled groups; there were some deep dimples mainly in the temporal region on spectral domain optical coherence tomography (SD-OCT) B-scan images in ILM-peeled eyes, but not in non-ILM-peeled eyes. In the color fundus photographs, a typical DONFL appearance ${ }^{19}$ was seen only in ILM-peeled eyes, and was seen over the entire macular area, especially in the area superior-temporal to the fovea. Dimples in the temporal macula, visible on SD-OCT horizontal B-scans, spatially corresponded to DONFL patterns, visible in both color fundus photography and three-dimensional SDOCT images. The dimples do not appear to be the result of damage caused by grasping the ILM, because it is unnatural that grasping would result in such a regular pattern.

The differences we found in this study were not necessarily large, compared to the mean retinal thickness of each subfield (241-322 $\mu \mathrm{m}$ for MH fellow eyes, and 254-329 $\mu \mathrm{m}$ for ERM fellow eyes). For MH surgery, when eyes with ILM peeling were compared to eyes without ILM peeling, mean macular thicknesses were significantly greater in the central (foveal) and inner nasal subfields (by $52 \mu \mathrm{m}$ and $26 \mu \mathrm{m}$, respectively), and significantly thinner in the inner temporal subfield (by $17 \mu \mathrm{m}$ ). The axial resolution of the SD-OCT instrument is $5 \mu \mathrm{m}$, which allows detection of small differences, particularly of thinning at the inner temporal subfield. Currently, it remains unknown as to which layer is most affected by ILM peeling. But we speculate that the inner retinal layers are most affected, based on observation of the SD-OCT B-scans (Figures 1 and 2). Single retinal layers, such as the retinal nerve fiber layer, ganglion cell layer, inner plexiform layer, and inner nuclear layer, are reportedly $33.9-37.5 \mu \mathrm{m}$ in mean thickness in normal eyes. ${ }^{30}$ Thus, when compared to the mean thicknesses of each single layer in normal eyes, differences, in both thicker and thinner regions, caused by ILM peeling, are not so small, possibly indicating the development of clinically significant changes in macula structures. This remains to be clarified.

Our results are consistent with those reported by Ohta et al, ${ }^{17}$ who studied the RT of the inner ring (with a radius of $1 \mathrm{~mm}$ ) and the outer ring (with a radius of $3 \mathrm{~mm}$ ), before and after vitrectomy with ILM peeling, in 10 eyes of 10 patients who had an idiopathic MH. Ohta et a $1{ }^{17}$ used the Spectralis ${ }^{\circledR}$ HRA-OCT (Heidelberg Engineering, Heidelberg, Germany) to measure RTs, and reported that the temporal retina was significantly thinner, and the nasal retina significantly thicker, in the parafoveal retina. Our study showed that region-specific changes in macular thickness were not confined to the parafoveal regions; in the MH-with-ILM peeling group, operated eyes had significantly thinner outer temporal macula and significantly thicker outer nasal macula. Our results further showed a thinner temporal macula and thicker nasal macula in eyes with ILM peeling, compared to those without ILM peeling. Furthermore, these regionspecific abnormalities after ILM peeling were seen in eyes after ERM surgery, and thus did not appear to be specific to eyes with MH.

Treumer et $\mathrm{al}^{14}$ reported that the nasal parafoveal retina, after ERM with ILM peeling, remained thickened for a mean follow-up time of $46 \pm 13$ months, while the thickness of other parafoveal areas, including the temporal parafovea, returned to normal. Although our study had a shorter follow-up time, and fellow eyes were used as controls, our results are consistent with their results; the nasal 

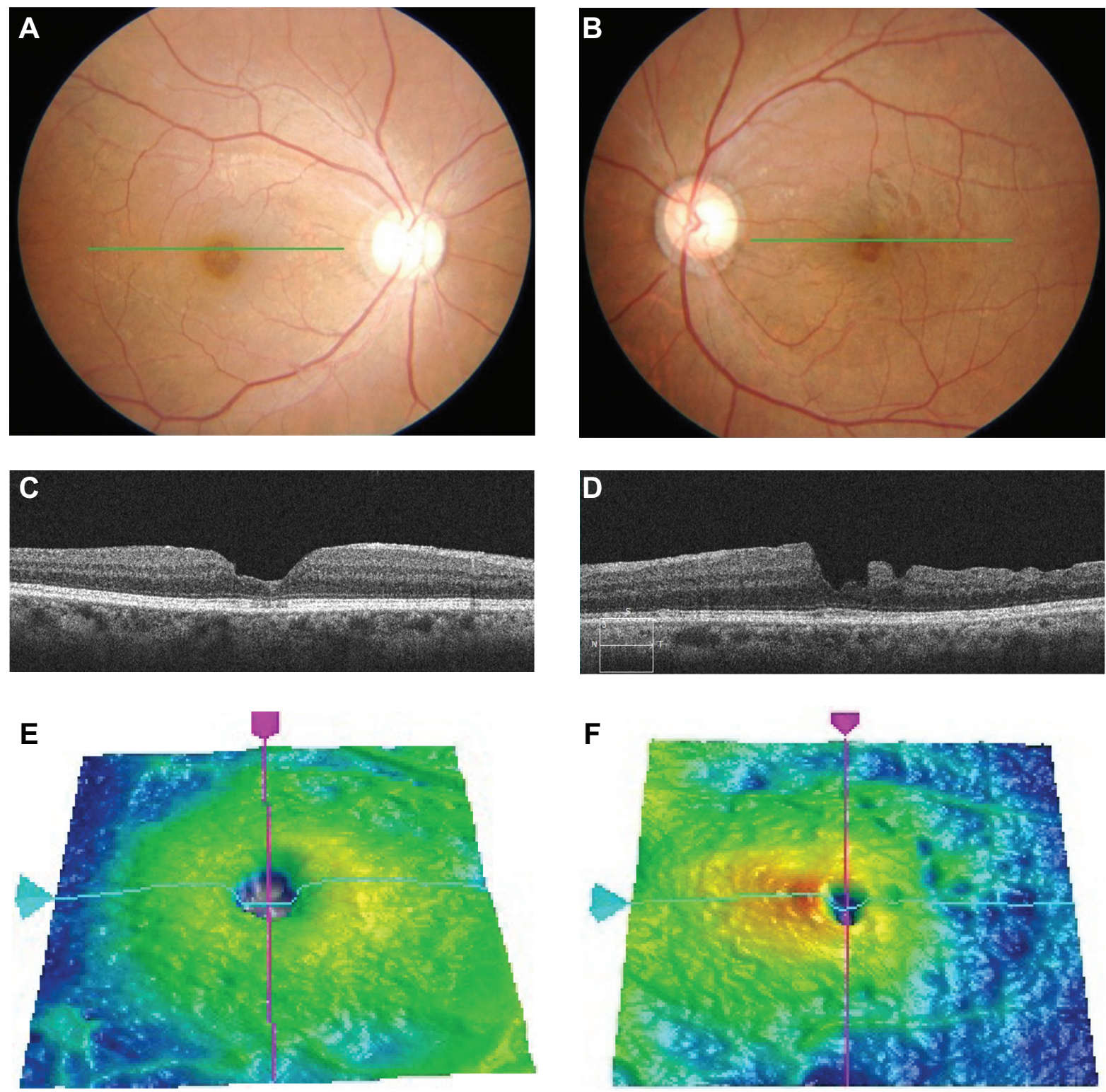

Figure I Color fundus photographs and Cirrus OCT images of an 82-year-old woman who underwent vitrectomy with and without ILM peeling for bilateral macular holes. Notes: (A) Fundus photograph of the right eye. The patient underwent vitrectomy without ILM peeling in I994. Visual acuity improved from 0.09 to 0.2. A DONFL appearance was not seen; (B) Fundus photograph of the left eye. The patient underwent vitrectomy with ILM peeling in 200 I. Visual acuity improved from 0.5 to I.0. A DONFL appearance was clearly visible; (C and E) Cirrus OCT image of a horizontal scan and 3-dimentional OCT image. The temporal to nasal ratio was 0.93; ( $\mathbf{D}$ and $\mathbf{F}$ ) Cirrus OCT image of a horizontal scan and 3-dimentional OCT image. The SD-OCT images had characteristic features in the temporal macula, such as an uneven surface of the retina and retinal thinning with abrupt depressions. The temporal to nasal ratio was 0.74 .

Abbreviations: OCT, optical coherence tomography; ILM, internal limiting membrane; DONFL, dissociated optic nerve fiber layer; SD-OCT, spectral domain optical coherence tomography.

parafoveal retina remained thickened, while the temporal parafovea returned to normal after ERM with ILM peeling. Our study showed that the superior and inferior regions also remained thickened, but the differences, compared to fellow eyes, were only $16 \mu \mathrm{m}$ for both, compared to $46 \mu \mathrm{m}$ for the nasal parafovea.

We also showed that the entire parafoveal area, including inner parafovea, remained thickened when an ILM was not peeled. Thus, it is possible that an inner parafovea, thinner than other parafoveal areas, long after ERM surgery, is also due to ILM peeling.

We previously used scanning laser ophthalmoscopy on 22 eyes, following surgery, with or without ILM peeling, for an idiopathic ERM. All eyes with ILM peeling $(n=10)$ had a disappearance of retinal folds, and three (30\%) of ten eyes showed many characteristic arcurate striae in the macular area. The striae were slightly darker than the surrounding retina and retinal nerve fibers. ${ }^{8}$ In the following study, ${ }^{18}$ 

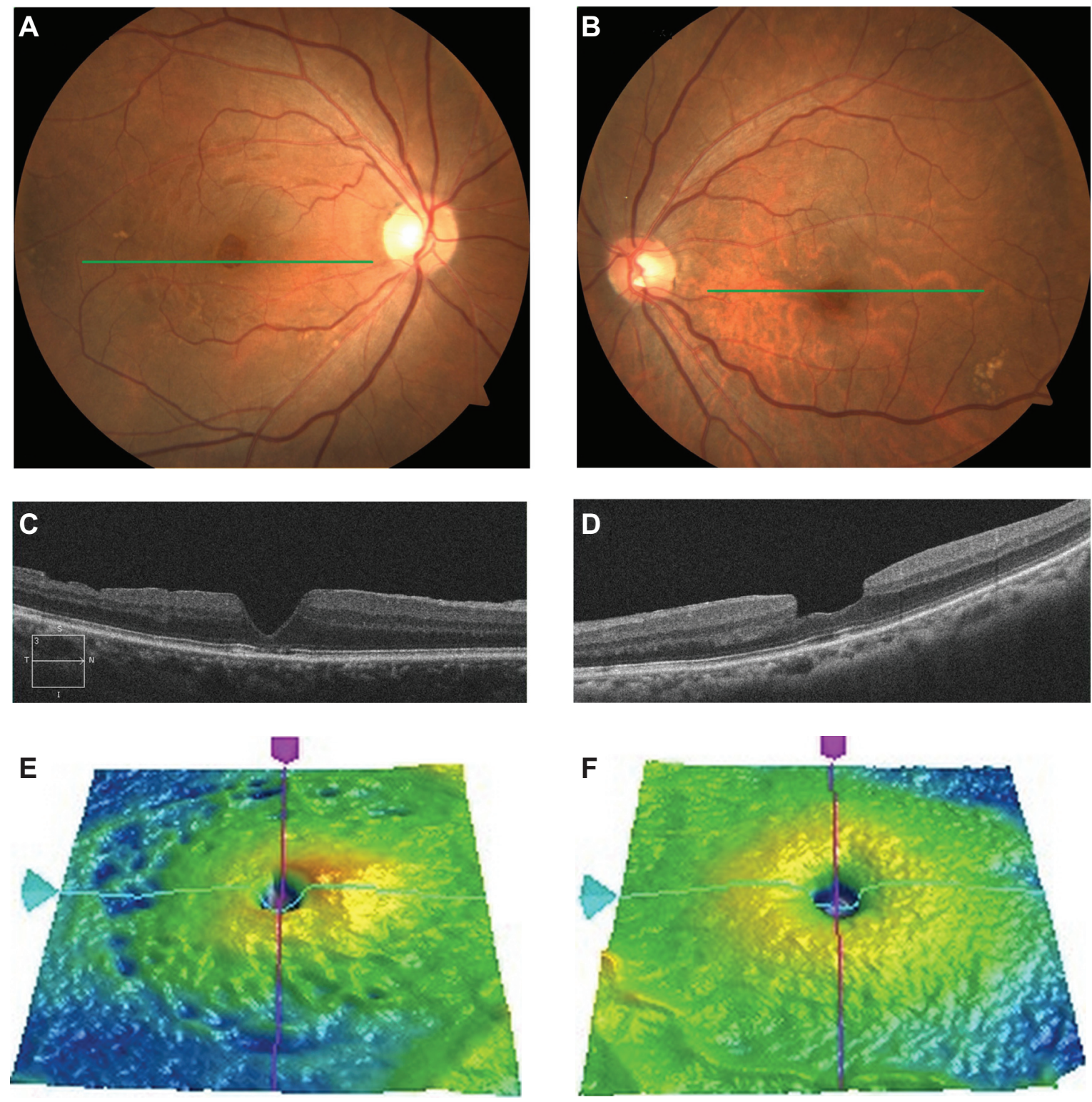

Figure 2 Color fundus photographs and Cirrus OCT images of an 80-year-old woman who underwent vitrectomy with and without ILM peeling for bilateral macular holes. Notes: (A) Fundus photograph of the right eye. The patient underwent vitrectomy with ILM peeling in 200I.Visual acuity improved from 0.3 to 0.8 . A DONFL appearance was clearly visible; (B) Fundus photograph of the left eye. The patient underwent vitrectomy without ILM peeling in I999.Visual acuity improved from 0.I to I.2. A DONFL appearance was not seen; (C and E) Cirrus OCT image of a horizontal scan. The SD-OCT images had characteristic features in the temporal macula, such as an uneven surface of the retina and retinal thinning with abrupt depressions. The temporal to nasal ratio was 0.88 ; (D and F) Cirrus OCT image of a horizontal scan. The temporal to nasal ratio was 0.96 .

Abbreviations: OCT, optical coherence tomography; ILM, internal limiting membrane; DONFL, dissociated optic nerve fiber layer; SD-OCT, spectral domain optical coherence tomography.

we showed that these characteristic fundus appearances were observed only in ILM-peeled eyes. The incidence was 12 out of 20 in MH surgery, and 14 out of 23 in ERM surgery.

Tadayoni et al ${ }^{19}$ were the first to call these striae "DONFL appearance". DONFL appearance is thought to be caused by depressions of the retina, limited to the thickness of the retinal nerve fiber layer. The mean depth was reported to be $28.6 \mu \mathrm{m} .{ }^{22}$ Thus, temporal retinal thinning may be caused, in part at least, by DONFL appearance. However, temporal retinal thinning was observed in eyes with and without DONFL appearance. In addition, all nine areas of eyes with DONFL appearance were thicker than those of eyes without it. Thus, the presence of DONFL appearance does not appear to be the direct cause of thinner temporal maculae in eyes that had MH surgery with ILM peeling. It remains to be determined whether the thicker maculae we observed in eyes with DONFL appearance may be responsible for the DONFL 
appearance, or whether the thicker maculae may be due to differences in the effects on the macula of ILM peeling.

ILM peeling significantly enhances the closure rate of macular holes. ${ }^{3-5}$ However, all patients with successful macular hole surgery do not show significant improvement in visual acuity, even when ILM peeling is performed. The results of our study cannot elucidate the reason for this. Christensen, ${ }^{5}$ in a randomized clinical trial involving 78 pseudophakic patients with $\mathrm{MH}$, compared the effects of indocyanine green- (ICG) assisted ILM peeling, trypan blue-assisted ILM peeling, and no ILM peeling on foveal morphology. They found that attenuation of the foveal photoreceptor layer, and disruption of the foveal photoreceptor inner and outer segment junction lines, were associated with poor visual acuity 12 months after successful macular hole surgery. Importantly, they also found that attenuation and disruption of the foveal photoreceptor layer was independent of ILM peeling or staining. The mean central retinal thickness was greater in eyes with ILM peeling than in eyes without ILM peeling, consistent with our results. But the central retinal thickness did not correlate with postoperative visual acuity. ${ }^{5}$ Thus, ILM peeling does not appear to have significant effects on postoperative foveal morphology, which is associated with postoperative visual acuity prognosis.

Although it is known that ICG-assisted ILM peeling causes visual field defects after macular hole surgery, it remains unclear whether it is only the use of ICG that is harmful, or whether the ILM peeling itself is also harmful. It is reported that small, mostly asymptomatic paracentral scotomata were found in more than half of eyes that received ILM peeling without ICG staining for macular hole surgery. ${ }^{31}$

The mechanisms by which ILM peeling results in thickening and thinning in different regions remain unknown. ILM is the basement membrane for Müller glial cells that support retinal structures longitudinally. Previous histopathological studies have shown that damage to Müller glial cells, following ILM peeling, may be responsible for the thinning in the temporal macula. ${ }^{32,33}$ However, in our study, both thickening and thinning occurred in the nasal and temporal macula, respectively. Damage to Müller glial cells does not appear to cause retinal thickening, at least 1 year after surgery. It is unknown whether only one mechanism can account for the complicated macular structural changes associated with ILM peeling. The most prominent anatomical difference between the nasal and temporal macula is the thickness of the retinal nerve fiber layer (RNFL). It is possible that the effects of ILM peeling reach the inner retina, deeper than the thin RNFL, in the temporal raphe, whereas they were limited to the thick
RNFL in the nasal macula. Further investigations are required to elucidate the actual adverse effects of ILM peeling.

Our study showed that the retina in the central area, in the MH-with-ILM peeling group, was significantly thicker than that of the non-ILM-peeled group. This finding is consistent with several reports. ${ }^{5,7,12,13,16}$ Christensen et al ${ }^{5,7}$ reported that surgical method did affect postoperative foveal thickness, which was significantly increased in ILM-peeled eyes, compared to non-peeled eyes. The microstructure of thickened retinas in ILM-peeled eyes was characterized by diffuse thickening of the inner retina, at the level of the outer nuclear layer, leading to flattening of the foveal pit. Non-peeled retinas were often characterized by deeper foveal pits, indicating that surgery with ILM peeling increased the mobility of the hole edges. ${ }^{5,7}$

Our study has several limitations, resulting from its retrospective nature. The number of cases of $\mathrm{MH}$ or ERM without ILM peeling is too small. This is because we routinely performed ILM peeling during the study period. Also, there was a difference in the preoperative severity of ERM between cases with and without ILM peeling. In this study, we compared the RT of operated eyes with fellow eyes in a relatively large number of $\mathrm{MH}$ or ERM cases, with ILM peeling, at least 12 month postoperatively.

It is possible that there was a selection bias in grouping our subjects. In macular hole surgeries, ILM peeling was performed after March 1998, and not performed before this time. Although selection bias by the surgeon appears to be minimal for the MH groups, there are still other biases, such as in the surgeon's learning curve for technique, and in refinement of vitreoretinal surgical instruments. In ERM surgery, ILM peeling was performed in a large proportion of eyes, and was not performed only in a small number of cases with weak retinal folds. Thus, selection bias by the surgeon occurred in the ERM groups.

Another limitation is the possibility that, in some ERM cases without ILM peeling, a part of the ILM might also have been peeled when the ERM was peeled. Histopathologic studies of extracted tissue specimens from surgically removed ERM often showed that ILM was present also. ${ }^{34}$ However, we still found significantly thinner retinas in the inner temporal area in ERM eyes that had ILM peeling, compared with those that did not receive ILM peeling.

\section{Conclusion}

The results of this study suggest that ILM peeling leads to a thinning of the temporal macula and thickening of the nasal macula. These region-specific changes in macular thickness 
do not appear to be disease-specific. Additional studies will be needed to determine whether region-specific changes in RT occur after ILM peeling, regardless of the disease, and whether these changes can cause any abnormalities in visual function.

\section{Disclosure}

The authors report no conflicts of interest in this work.

\section{References}

1. Eckardt C, Eckardt U, Groos S, Luciano L, Reale E. Removal of the internal limiting membrane in macular holes. Clinical and morphological findings. Ophthalmologe. 1997;94:545-551.

2. Olsen TW, Sternberg P Jr, Capone A Jr, et al. Macular hole surgery using thrombin-activated fibrinogen and selective removal of the internal limiting membrane. Retina. 1998;18:322-329.

3. Brooks HL Jr. Macular hole surgery with and without internal limiting membrane peeling. Ophthalmology. 2000;107:1939-1948.

4. Kumagai K, Furukawa M, Ogino N, Uemura A, Demizu S, Larson E. Vitreous surgery with and without internal limiting membrane peeling for macular hole repair. Retina. 2004;24:721-727.

5. Christensen UC. Value of internal limiting membrane peeling in surgery for idiopathic macular hole and the correlation between function and retinal morphology. Acta Ophthalmol. 2009;87 Thesis 2:1-23.

6. Kumagai K, Furukawa M, Ogino N, Larson E. Incidence and factors related to macular hole reopening. Am J Ophthalmol. 2010;149: 127-132.

7. Christensen UC, Kroyer K, Sander B, Jorgensen TM, Larsen M, la Cour M. Macular morphology and visual acuity after macular hole surgery with or without internal limiting membrane peeling. Br J Ophthalmol. 2010;94:41-47.

8. Ishikawa F, Ogino N, Hirane M, et al. Scanning laser ophthalmoscopic findings following peeling of internal limiting membrane for idiopathic epiretinal membrane. Jpn J Clin Ophthalmol. 2000;54:1693-1696.

9. Park DW, Dugel PU, Garda J, et al. Macular pucker removal with and without internal limiting membrane peeling: pilot study. Ophthalmology. 2003;110:62-64.

10. Kwok AKh, Lai TY, Yuen KS. Epiretinal membrane surgery with or without internal limiting membrane peeling. Clin Experiment Ophthalmol. 2005;33:379-385.

11. Kumagai K, Ogino N, Demizu S, Atsumi K, Kurihara H, Ishigooka H. Peeling of internal limiting membrane for macular pseudoholes. Jpn J Clin Ophthalmol. 2002;56:361-367.

12. Okita K, Ogino N, Shirai M, et al. Internal limiting-membrane peeling for idiopathic epiretinal membrane: one-year course of visual acuity, retinal sensitivity and foveal thickness. Atarashii Ganka. 2000; 17:1437-1440

13. Lee JW, Kim IT. Outcomes of idiopathic macular epiretinal membrane removal with and without internal limiting membrane peeling: a comparative study. Jpn J Ophthalmol. 2010;54:129-134.

14. Treumer F, Wacker N, Junge O, Hedderich J, Roider J, Hillenkamp J. Foveal structure and thickness of retinal layers long-term after surgical peeling of idiopathic epiretinal membrane. Invest Ophthalmol Vis Sci. 2011;52:744-750.

15. Terasaki H, Miyake Y, Nomura R, et al. Focal macular ERGs in eyes after removal of macular ILM during macular hole surgery. Invest Ophthalmol Vis Sci. 2001;42:229-234.

16. Kumagai K, Furukawa M, Ogino N, Larson E. Possible effects of internal limiting membrane peeling in vitrectomy for macular vein occlusion. Jpn J Ophthalmol. 2010;54:61-65.
17. Ohta K, Sato A, Fukui E. Asymmetrical thickness of parafoveal retina around surgically closed macular hole. Br J Ophthalmol. 2010;94: 1545-1546.

18. Ishikawa F, Ogino N, Okita K, et al. Retinal nerve fiber layer defect after vitrectomy and internal limiting membrane peeling for idiopathic macular hole and epiretinal membrane. Jpn J Clin Ophthalmol. 2001;55:1539-1544.

19. Tadayoni R, Paques M, Massin P, et al. Dissociated optic nerve fiber layer appearance of the fundus after idiopathic epiretinal membrane removal. Ophthalmology. 2001;108:2279-2283.

20. Tamura K, Matsui Y, Iwao K, et al. Findings simulating retinal nerve fiber layer defect following peeling of internal limiting membrane. Jpn J Clin Ophthalmol. 2002;56:1601-1605.

21. Tamura K, Matsui Y, Sugimoto T, et al. Long term course of findings simulating dissociated optic nerve fiber layer following peeling of internal limiting membrane. Jpn J Clin Ophthalmol. 2004;58:381-385.

22. Mitamura Y, Suzuki T, Kinoshita T, et al. Optical coherence tomographic findings of dissociated optic nerve fiber layer appearance. Am J Ophthalmol. 2004;137:1155-1156.

23. Mitamura Y, Ohtsuka K. Relationship of dissociated optic nerve fiber layer appearance to internal limiting membrane peeling. Ophthalmology. 2005; 112:1766-1770.

24. Ito Y, Terasaki H, Takahashi A, Yamakoshi T, Kondo M, Nakamura M. Dissociated optic nerve fiber layer appearance after internal limiting membrane peeling for idiopathic macular holes. Ophthalmology. 2005; 112:1415-1420.

25. Imai $\mathrm{H}$, Ohta $\mathrm{K}$. Microperimetric determination of retinal sensitivity in areas of dissociated optic nerve fiber layer following internal limiting membrane peeling. Jpn J Ophthalmol. 2010;54:435-440.

26. Kumagai K, Hangai M, Larson E, Ogino N. Vitreoretinal interface and foveal deformation in asymptomatic fellow eyes of patients with unilateral macular holes. Ophthalmology. 2011;118:1638-1644.

27. Kimura H, Kuroda S, Nagata M. Triamcinolone acetonide-assisted peeling of the internal limiting membrane. Am J Ophthalmol. 2004;137: 172-173.

28. Early Treatment Diabetic Retinopathy Study Research Group. Grading diabetic retinopathy from stereoscopic color fundus photographs - an extension of the modified Airlie House classification. ETDRS report number 10. Ophthalmology. 1991;98:786-806.

29. Ooto S, Hangai M, Sakamoto A, et al. Three-dimensional profile of macular retinal thickness in normal Japanese eyes. Invest Ophthalmol Vis Sci. 2010;51:465-473.

30. Tan O, Li G, Lu AT, Varma R, Huang D; Advanced Imaging for Glaucoma Study Group. Mapping of macular substructures with optical coherence tomography for glaucoma diagnosis. Ophthalmology. 2008;115:949-956.

31. Haritoglou C, Gass CA, Schaumberger M, Ehrt O, Gandorfer A, Kampik A. Macular changes after peeling of the internal limiting membrane in macular hole surgery. Am J Ophthalmol. 2001;132: 363-368.

32. Wolf S, Schnurbusch U, Wiedemann P, Grosche J, Reichenbach A, Wolburg H. Peeling of the basal membrane in the human retina: ultrastructural effects. Ophthalmology. 2004;111:238-243.

33. Nakamura T, Murata T, Hisatomi T, et al. Ultrastructure of the vitreoretinal interface following the removal of the internal limiting membrane using indocyanine green. Curr Eye Res. 2003;27:319-320.

34. Okada M, Ogino N, Matsumura M, Honda Y, Nagai Y. Histological and immunohistochemical study of idiopathic epiretinal membrane. Ophthalmic Res. 1995;27:118-128. 


\section{Publish your work in this journal}

Clinical Ophthalmology is an international, peer-reviewed journal covering all subspecialties within ophthalmology. Key topics include: Optometry; Visual science; Pharmacology and drug therapy in eye diseases; Basic Sciences; Primary and Secondary eye care; Patient Safety and Quality of Care Improvements. This journal is indexed on
PubMed Central and CAS, and is the official journal of The Society of Clinical Ophthalmology (SCO). The manuscript management system is completely online and includes a very quick and fair peer-review system, which is all easy to use. Visit http://www.dovepress.com/ testimonials.php to read real quotes from published authors. 